Nota Científica

\title{
Contribución al conocimiento de la distribución geográfica de Euchroma gigantea (Linnaeus, 1758) (Coleoptera: Buprestidae) en Venezuela
}

\author{
Contribution to the knowledge of the geographical distribution of Euchroma gigantea \\ (Linnaeus, 1758) (Coleoptera: Buprestidae) in Venezuela
}

Joffre Blanco $^{1}$ (D) ,Evelin Arcaya Sánchez $^{2 *}$ (D) Raffaele Acconcia $^{3}$

\begin{abstract}
${ }^{1}$ Laboratorio de Entomología, Universidad Nacional Experimental del Táchira. San Cristóbal, estado Táchira, Venezuela. ${ }^{2}$ Departamento de Ciencias Biológicas. Decanato de Agronomía. Universidad Centroccidental "Lisandro Alvarado" (UCLA). Lara, Venezuela. 隐evearcaya@gmail.com. ${ }^{3}$ Fundación Entomológica Andina, quinta Mi Ranchito, calle Urdaneta, sector Manzano Bajo, Ejido, Estado Mérida, Venezuela.
\end{abstract}

\section{ZooBank: urn:lsid:zoobank.org:pub:93A42BFD-D1A1-4A75-A204-520AF33EAEA8 https: / / doi.org/10.35249/rche.47.2.21.03}

Resumen. Euchroma gigantea (Linnaeus, 1758) o "Barrenador gigante metálico de la Ceiba", es una especie de Buprestidae (Insecta: Coleoptera) distribuida en gran parte de América. El presente trabajo se realiza con la finalidad de ampliar el conocimiento de la distribución geográfica de E. gigantea en Venezuela. Con la información recopilada aumenta el número de estados registrados para el país, además se incrementan los datos de distribución geográfica local y a nivel del continente americano.

Palabras clave: Chrysochroinae; Malvaceae; Neotrópico; nuevos registros; Sudamérica.

Abstract. Euchroma gigantea (Linnaeus, 1758) or "Giant metallic borer of the Ceiba", is a species of Buprestidae (Insecta: Coleoptera) distributed in much of America. The present work is carried out in order to expand the knowledge of the geographical distribution of E. gigantea in Venezuela. With the information collected, the number of states registered for the country increases, as well as the local geographic distribution data and at the level of the American continent.

Key words: Chrysochroinae; Malvaceae; Neotropics; new records; South America.

Euchroma gigantea (Linnaeus, 1758) conocida como el "Barrenador gigante metálico de la Ceiba", es una especie de Buprestidae (Insecta: Coleoptera) distribuida ampliamente en América y la única representante del género Euchroma Dejean, 1833. Esta llamativa especie es considerada la de mayor tamaño entre los bupréstidos americanos (Bellamy 2010).

La clasificación actualmente válida de E. gigantea la ubica dentro de las cinco subespecies descritas (i.e., E. g. gigantea (Linnaeus, 1758), E. g. goliath (Laporte y Gory, 1836), E. g. harperi Sharp, 1881, E. g. inca Obenberger, 1928 y E. g. peruana Obenberger, 1928) que habitan la región Neotropical (Blackwelder 1944; Moura et al. 2008; Bellamy 2008). En Venezuela, están presentes las subespecies E. g. gigantea y E. g. goliath. La subespecie E. g. gigantea se distribuye en los estados Amazonas, Barinas, Bolívar, Delta Amacuro, Guárico, Portuguesa, Sucre y Táchira, mientras que E. g. goliath se encuentra

Recibido 6 Marzo 2021 / Aceptado 29 Marzo 2021 / Publicado online 23 Abril 2021

Editor Responsable: José Mondaca E. 
en Bolívar, Carabobo y Distrito Federal (Hornburg 2014).

Los adultos de E. gigantea pueden llegar a medir $8 \mathrm{~cm}$ de longitud y hasta $2,5 \mathrm{~cm}$ de ancho, presentan una coloración corporal verde metálico con un tinte rojizo o violáceo en los élitros y dos grandes manchas negruzcas en el pronoto. La superficie elitral tiene una textura rugosa y está cubierta por una pulverulencia cerosa amarillenta caediza en los adultos recién emergidos (Hogue 1993; Boppré 2003; Rosado y Salazar 2005). Las larvas también de gran tamaño alcanzan una longitud de 12-15 cm (García 1998), poseen un cuerpo alargado con la cabeza amarillenta y un tórax en forma de disco aplanado. Los estados inmaduros de esta especie se encuentran habitualmente dentro de la madera blanda de árboles pertenecientes a la familia Malvaceae (e.g., ceiba gigante (Ceiba pentandra (L.) Gaertn.), balsa (Ochroma spp.), castaño de agua (Pachira aquatica Aubl.) y pseudobombax (Pseudobombax spp.)), así como de las familias Araucariaceae (pino Paraná (Araucaria angustifolia (Bertol.) Kuntze)) y Moraceae (higuera (Ficus abelii Miq.)) (Hogue 1993; Boppré 2003; Rodrigues-Netto et al. 2003; Rosado y Salazar 2005).

Euchroma gigantea se distribuye desde el sur de los Estados Unidos de América hasta el sur de Sudamérica en regiones con clima cálido seco o húmedo hasta los $1200 \mathrm{~m}$ de altitud (Rosado y Salazar 2005). En Brasil ha sido registrada en el Distrito Federal y en los estados de Sao Paulo, Goiás, Pará, Pernambuco, Bahía y Alagoas (Silva et al. 1968; Fonseca 2010). También, está presente en países como Argentina, Belice, Bolivia, Colombia, Costa Rica, Ecuador, Guatemala, Guayana Francesa, Guyana, Honduras, Jamaica, México, Nicaragua, Panamá, Paraguay, Perú y Surinam (Ramos-Elorduy y Pino-Moreno 2004; Rosado y Salazar 2005; Arguedas 2006; Álvarez et al. 2014; GBIF 2019; da Silva y Félix 2020; Oubter et al. 2020; Hashimoto 2021; Ueda 2021).

En Venezuela esta especie ha sido reportada en los estados Aragua (Osuna 2000), Amazonas, Barinas, Bolívar, Carabobo, Delta Amacuro, Distrito Federal, Guárico, Portuguesa, Sucre y Táchira (Hornburg 2014).

Con base al contexto anterior, la presente nota se realiza con la finalidad de contribuir al conocimiento de la distribución geográfica de E. gigantea en Venezuela. Para esto se revisaron los ejemplares de E. gigantea depositados en las colecciones de Museo Entomológico José Manuel Osorio (MJMO) de la Universidad Centrooccidental Lisandro Alvarado (UCLA), estado Lara, Fundación Entomológica Andina (CFUNEA), estado Mérida, y colección privada de Joffre Blanco (CJB), estado Táchira. Para determinar la distribución geográfica de la especie se recopilaron las localidades de recolección de los ejemplares estudiados. Estos lugares fueron georreferenciados usando Google Earth Pro (v7.3.2.5776) y los datos obtenidos ingresados al programa online SimpleMappr (Shorthouse 2010) para generar el mapa de distribución.

Las imágenes de los especímenes ilustrados fueron obtenidas con una cámara digital marca Canon modelo EOS Rebel T4i®. Los ejemplares fotografiados pertenecen a CJB.

Se registraron 47 ejemplares de E. gigantea (23 machos y 24 hembras), los cuales fueron recolectados en varios lugares de Venezuela. Los estados con mayor número de especímenes fueron Lara (18), Yaracuy (10) y Barinas (5), los que en su conjunto representan el $70 \%$ de los ejemplares de esta especie depositados en colecciones entomológicas. El mapa obtenido muestra la distribución espacial de E. gigantea en territorio venezolano (Fig. 1), específicamente en los estados Amazonas, Falcón, Aragua, Portuguesa, Mérida y Táchira. Al respecto, Osuna (2000) la citó previamente para el estado Aragua, mientras que Hornburg (2014) sitúa a esta especie en los estados Amazonas, Barinas, Bolívar, Delta Amacuro, Guárico, Portuguesa, Sucre y Táchira. Los nuevos registros obtenidos permiten aumentar el número de estados con presencia de E. gigantea en Venezuela, según el siguiente detalle: 


\section{Euchroma gigantea (Linnaeus, 1783)}

(Figs. 1, 2)

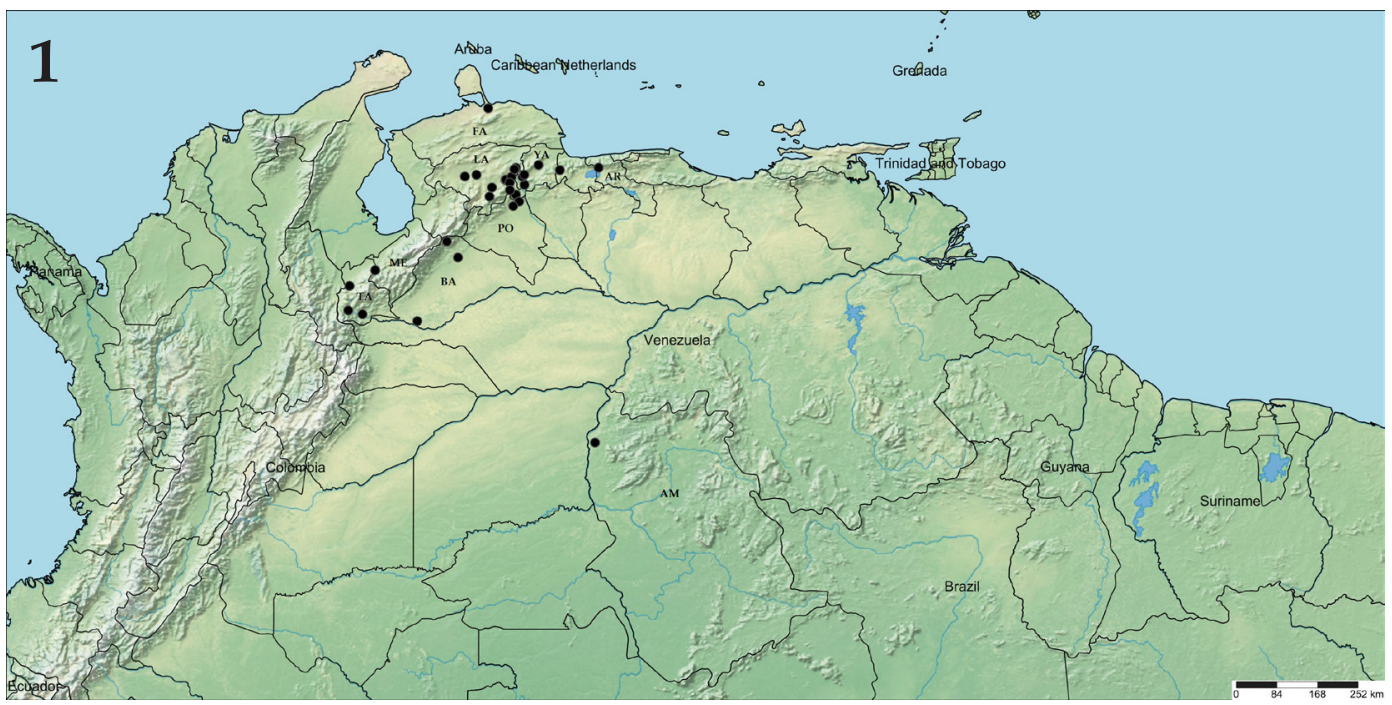

Figura 1. Distribución geográfica de Euchroma gigantea gigantea (Linnaeus, 1783) en Venezuela. Los estados representados son: Amazonas (AM), Aragua (AR), Barinas (BA), Falcón (FA), Lara (LA), Mérida (ME), Portuguesa (PO), Táchira (TA) y Yaracuy (YA). / Geographical distribution of Euchroma gigantea (Linnaeus, 1783) in Venezuela. The states represented are: Amazonas (AM), Aragua (AR), Barinas (BA), Falcón (FA), Lara (LA), Mérida (ME), Portuguesa (PO), Táchira (TA) and Yaracuy (YA).
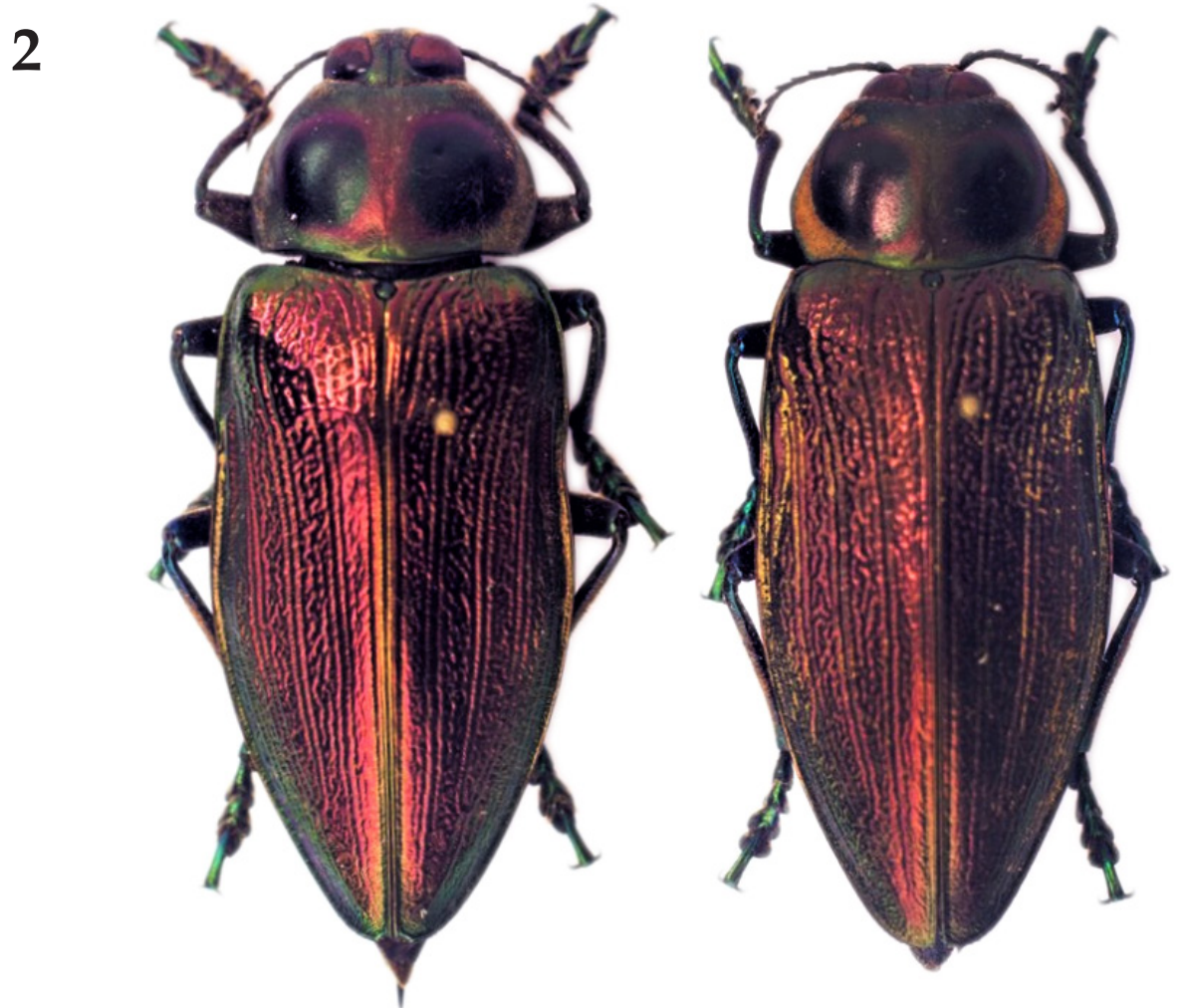

Figura 2. Vista dorsal de Euchroma gigantea (Linnaeus, 1783). 1. Macho. 2. Hembra. Escala: $1 \mathrm{~cm} . /$ Dorsal view of Euchroma gigantea (Linnaeus, 1783). 1. Male. 2. Female. Scale: $1 \mathrm{~cm}$. 
Material examinado. Venezuela. Estado Amazonas: $1 q$ (MJMO). Porvenir-Cejal, municipio Atures, $91 \mathrm{msnm}, 5^{\circ} 11^{\prime} 07.17^{\prime \prime} \mathrm{N}, 67^{\circ} 41^{\prime} 43.66^{\prime \prime} \mathrm{O}, 1-\mathrm{XII}-198$, W. Olivo leg. Estado Aragua: 1 ㅇ (MJMO). El Limón, municipio Mario Briceño Iragorry, $450 \mathrm{msnm}, 10^{\circ} 17^{\prime} 25.48^{\prime \prime} \mathrm{N}$, 67³7’38.92”O, 10-V-1985, F. Díaz leg. 1ð^ (CJB). El Limón, municipio Mario Briceño Iragorry, 450 msnm, 10¹7'25.48' N, 67³7'38.92”'O, 3-IX-2009, A. Orellana leg. Estado

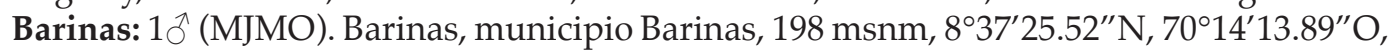
1-XII-1991, M. Alvarado leg. $1 \delta^{\jmath}$ (CFUNEA). Finca Valle Encantado, sector San Ramón, Calderas de Barinas, municipio Bolívar, $1104 \mathrm{msnm}, 8^{\circ} 55^{\prime} 02.72^{\prime \prime} \mathrm{N}, 70^{\circ} 26^{\prime} 46.37^{\prime \prime} \mathrm{O}$, VII2006, R. Acconcia leg. [recolectado posado sobre tronco en descomposición a primeras horas de la mañana, el área corresponde a fragmento de selva secundaria de selva nublada de pie de monte andino llanero]. 19 (CFUNEA). Reserva Forestal Caparo, Pica 8, municipio Andrés Eloy Blanco, 159 msnm, 7²6’42.56”N, 7059’45.12”O, 24-VIII-2014, R. Acconcia y J. Gámez leg. [recolectado en horas de la mañana sobre árbol talado, el área corresponde a bosques semideciduos]. 1 ㅇ (CFUNEA). Finca Valle Encantado, sector San Ramón, Calderas de Barinas, municipio Bolívar, 1263 msnm, 855’02.72”'N, 70²6'46.37"O, 24-V-2017, R. Acconcia leg. [recolectado sobre rama de árbol, el área corresponde a bosque pluvial mesotérmico o nublado]. 19 (CFUNEA). Finca Valle Encantado, sector San Ramón, Calderas de Barinas, municipio Bolívar, 1179 msnm, 855’02.72”N , 70²6’46.37”O, 22-VI2018, E. Albarrán leg. [recolectado alrededor de vivienda, el área corresponde a bosque pluvial mesotérmico o nublado]. Estado Falcón: 1 ( $\mathrm{MJMO})$. Coro, municipio Miranda, 22 msnm, 11 ${ }^{\circ} 3^{\prime} 45.3^{\prime \prime} \mathrm{N}, 6^{\circ} 40^{\prime} 48.25^{\prime \prime} \mathrm{O}, 18-\mathrm{V}-1991$, J. Infante leg. Estado Lara: $1 q$ (MJMO). Barquisimeto, sector Obelisco-UCLA, municipio Iribarren, $600 \mathrm{msnm}, 10^{\circ} 03^{\prime} 59.79^{\prime \prime} \mathrm{N}$, 69²1'34.76"O, 6-III-1978, I. Martínez leg. 4ð (MJMO). Barquisimeto, municipio Iribarren, 590 msnm, 1004'03.98'N, 69²0'50.46"O, varios datos: 2-IV-1978, L. Hernández leg., 10-

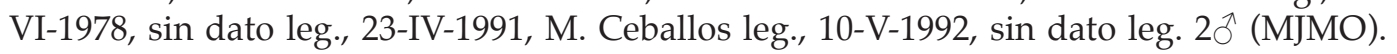
Cabudare, municipio Palavecino, $481 \mathrm{msnm}, 10^{\circ} 01^{\prime} 25.45^{\prime \prime} \mathrm{N}, 69^{\circ} 16^{\prime} 14.23^{\prime \prime} \mathrm{O}$, varios datos: 9-VII-1992, A. Escalona leg., 27-VI-2012, J. Blanco leg. $1 \hat{\jmath}$ (MJMO). Duaca, municipio

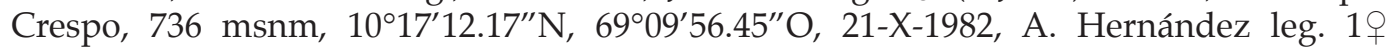
(MJMO). El Cercado, municipio Iribarren, 529 msnm, 1007'10.03” N, 69¹4'45.72"O, 12V-2020, M. Leo y A. Sánchez leg. 1ð̋ (MJMO). El Eneal, municipio Crespo, 700 msnm, $10^{\circ} 15^{\prime} 49.65^{\prime \prime} \mathrm{N}, 6^{\circ} 11^{\prime} 30.28^{\prime \prime} \mathrm{O}, 15-\mathrm{II}-1991$, R. Arias leg. 20; 1 q (MJMO). Las Palmitas, municipio Torres, $435 \mathrm{msnm}, 10^{\circ} 07^{\prime} 48.95^{\prime \prime} \mathrm{N}, 70^{\circ} 06^{\prime} 46.44^{\prime \prime} \mathrm{O}, 15-\mathrm{IX}-1975, \mathrm{~N}$. Pérez leg. $1{ }^{\jmath}$ (MJMO). Quíbor, municipio Jiménez, 700 msnm, 955'26.20”N, 69³6'28.96”'O, 15-VI1975, N. Hernández leg. 1 q (MJMO). Sanare, municipio Andrés Eloy Blanco, 1341 msnm, 945’12.96"N , 69³9’04.48”O, 23-VI-1991, M. Espósito leg. $1 \overbrace{}^{\prime \prime}$ (MJMO). Sarare, municipio Simón Planas, 289 msnm, 947'22.21”N , 6909'56.29”O, 2-III-1991, M. Rivas leg. 1ठ (MJMO). Tarabana, Decanato de Agronomía-UCLA, municipio Palavecino, $560 \mathrm{msnm}$, $10^{\circ} 00^{\prime} 58.51^{\prime \prime} \mathrm{N}, 6^{\circ} 16^{\prime} 59.47^{\prime \prime} \mathrm{O}, 14-\mathrm{V}-1991$, L. Gruber leg. 19 (MJMO). Parque Nacional Terepaima, municipio Palavecino, 1244 msnm, 952'21.66” N, 69¹6'33.63"O, 10-III-1991, J. Méndez leg. Estado Mérida: $1 q$ (MJMO). Mérida, municipio Libertador, 1375 msnm, 9³9’48.05”N , 6906'01.50”O, 9-III-1973, F. Gutiérrez y R. González leg. 10̊; 2 q (CFUNEA). Finca Campo Alegre, sector Palmarito, municipio Zea, $624 \mathrm{msnm}, 8^{\circ} 23^{\prime} 07.55^{\prime \prime} \mathrm{N}$, $71^{\circ} 46^{\prime} 33.24^{\prime \prime} \mathrm{O}$, varios datos: 1 , , V-2000, R. Acconcia leg. [recolectado sobre árbol talado de Balso Ochroma sp., Malvaceae, en horas del mediodía, el área corresponde a zona agrícola en selva húmeda submontana], 1§ર, IX-2014, R. Acconcia leg. [recolectado posado en horas de la mañana sobre rama de Ceiba Ceiba pentandra Malvaceae, el área corresponde a zona agrícola en selva húmeda submontana], 1, 13-V-2017, R. Acconcia leg. [recolectado sobre árbol de Balso Ochroma sp., Malvaceae en descomposición después del medio día, el área corresponde a zona agrícola en selva húmeda submontana]. Estado Portuguesa: $1+$ (MJMO). Agua Blanca, municipio Agua Blanca, 176 msnm, 9³9'48.83"N , 6906'01.97"O, 15-III-1992, sin dato leg. 1q (MJMO). Araure, municipio Araure, 206 msnm, 9³4'49.10”N, 
69¹2'58.59”'O, 12-II-1982, P. Rodríguez leg. Estado Táchira: 19 (CJB). San Félix carretera Colón, La Fría, municipio Ayacucho, 510 msnm, 805'59.41”N , 72¹5’04.49”'O, 20-II-2010, J. Blanco leg. $1 q$ (CJB). Santa Ana del Táchira, municipio Córdoba, 805 msnm, 7³8’36.73” N, 72¹6'40.01"O, V-2004, J. Blanco leg. 2`̃ (CJB). Santo Domingo El Piñal, Hacienda Santa Rosa, municipio Fernández Feo, 342 msnm, 7³4’07.62”N, 7200’56.46"O, varios datos:

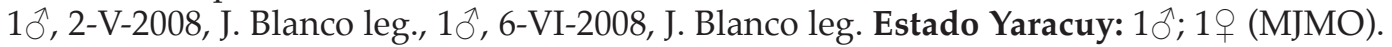
Chivacoa, municipio Bruzual, 303 msnm, 1009'26.06”' N, 6953'50.38' O, varios datos: 1 우, 4-VII-1982, H. Soto leg., 1ठ, 19-IX-1983 H. Soto leg. 1ð (MJMO). Las Velas, municipio Peña, 336 msnm, 958'24.50”N , 690'07.53”O, 17-XI-2011, J. Barradas leg. 2ㅇ (MJMO). Quebrada Seca, municipio Bolívar, 132 msnm, 10³2'57.39”N, 651'56.87”O, 20-III-1992, sin dato leg. 1 ㅇ (MJMO). Sabana de Parra, municipio José Antonio Páez, 419 msnm, $10^{\circ} 07^{\prime} 10.93^{\prime \prime} \mathrm{N}, 69^{\circ} 02^{\prime} 21.50^{\prime \prime} \mathrm{O}, 20-\mathrm{XI}-2000$, E. Carrasquero leg. 10̄ (MJMO). San Felipe, municipio San Felipe, 298 msnm, 10²0'27.33"N N, 6844'42.95”'O, 2-VIII-1992, A. Escalona leg. $20^{\top}$ (MJMO). San Pablo, municipio Arístides Bastidas, 365 msnm, 10¹4’57.52”N,

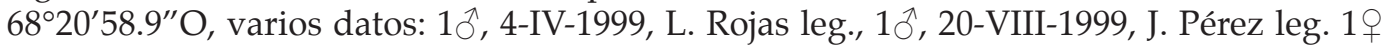
(MJMO). Urachiche, municipio Urachiche, $488 \mathrm{msnm}, 10^{\circ} 09^{\prime} 33.22^{\prime \prime} \mathrm{N}, 69^{\circ} 00^{\prime} 31.45^{\prime \prime} \mathrm{O}$, 26VIII-1999, C. Anselmo leg.

\section{Conclusiones}

Con base en el material examinado, se amplía la distribución geográfica de E. gigantea en Venezuela, incorporando cuatro nuevos estados situados en las regiones Centro Occidental (Falcón, Lara y Yaracuy) y Andina (Mérida) del país. Debido a la cercanía con los estados antes mencionados, es muy probable que esta especie también se localice en los estados Apure y Zulia.

\section{Agradecimientos}

Al Dr. Santos Rojo de la Universidad de Alicante (Alicante, España) y al Profesor Yohan Solano del Decanato de Agronomía de la Universidad Centroccidental Lisandro Alvarado (estado Lara, Venezuela) por el apoyo en la logística para la realización del trabajo. A Manuel García (Universidad Nacional Experimental del Táchira), por las fotografías de E. gigantea y al Ing. Guillermo González (Santiago, Chile), por su colaboración en el perfeccionamiento de la imagen de los ejemplares de E. gigantea. A los revisores anónimos por sus valiosos comentarios y sugerencias para mejorar nuestro trabajo.

\section{Literatura Citada}

Álvarez García, D.M., Arroyo Pérez, W.Y. y Navarro Iriarte, L.E. (2014) Report of Euchroma gigantea (Linnaeus, 1758) (Coleoptera: Buprestidae) for three departments of Colombian Caribbean Region. Acta Zoológica Mexicana, 30(3): 727-730.

Arguedas, L. (2006) Clasificación de tipos de daños producidos por insectos forestales. Segunda parte. Kurú: Revista Forestal, 3: 64-71.

Bellamy, C.L. (2008) A World Catalogue and Bibliography of the Jewel Beetles (Coleoptera: Buprestoidea). Volume 1: Introduction; Fossil Taxa; Schizopodidae; Buprestidae: Julodinae - Chrysochroinae: Poecilonotini. Pensoft Series Faunistica No. 76, 625 pp. Pensoft Publishers, Sofia-Moscow.

Bellamy, C.L. (2010) The world of jewel beetles - world catalogue. Family Buprestidae Leach, 1815. Consultado 23 febrero 2021. Disponible en: https:// cerambycids.com/ buprestidae/WorldCat/Classif/ webcat.html 
Blackwelder, R.E. (1944) Checklist of the coleopterous insects of Mexico, Central America, The West Indies, and South America. Bulletin of the United States National Museum, 185: 189-341.

Boppré, M. (2003) Roland Mecke, Insects of the Brazilian Pine. Studies on Neotropical Fauna and Environment, 38(2): 155.

da Silva Soares Souto, A. y Felix, M. (2020) Fiocruz/CEIOC - Coleção Entomológica do Instituto Oswaldo Cruz. Version 1.50. FIOCRUZ - Oswaldo Cruz Foundation. Occurrence dataset. Consultado 20 febrero 2021. https:// doi.org/10.15468/bdv6wn acceso vía GBIF.org. https: / / www.gbif.org/ occurrence/1457244780

Fonseca, A.P. (2010) Aspectos biológicos de Euchroma gigantea (Linnaeus, 1758) (Coleoptera: Buprestidae) em Pachira aquática Aubl. (1775) (Bombacaceae). Tesis de Maestría. Universidade Federal de Alagoas. Río Largo, Alagoas, Brasil. 100 pp.

García, A. (1998) Aspectos sobre a biología de Euchroma gigantea (L., 1758) (Coleoptera: Buprestidae) em Paquira acuatica Aublet (Bombacaceae). Pesquisa Agropecuária Tropical, 28: 69-73.

GBIF (The Global Biodiversity Information Facility) (2019) Euchroma giganteum (Linnaeus, 1758) en GBIF Secretariat (2019). GBIF Backbone Taxonomy. Checklist dataset. Consultado 20 febrero 2021. https: / / doi.org / 10.15468/39omei

Hashimoto, Y. (2021) EntomologicalSpecimens of Museum of Nature and Human Activities, Hyogo Pref., Japan. Version 2.3. National Institute of Genetics, ROIS. Occurrence dataset. Consultado 20 febrero 2021. Disponible en: https: / / doi.org/10.15468/bqkybh accesso vía GBIF. org https: / / www.gbif.org/occurrence/768165326

Hogue, C.L. (1993) Latin American Insects and Entomology. University of California Press, Berkeley and Los Angeles, California. 521 pp.

Hornburg, M. (2014) Buprestid Beetles of Venezuela. Consultado 25 enero 2021. Disponible en: http:/ / buprestidae.de/content/E_giganteum.html

Moura, R.C., Melo, N.F. y Souza, M.J. (2008) High levels of chromosomal differentiation in Euchroma gigantea L. 1735 (Coleoptera, Buprestidae). Genetics and Molecular Biology, 31(2): 431-437.

Osuna, E. (2000) Entomología del Parque Nacional Henri Pittier Aragua. Venezuela. Ediciones Fundación Polar. Caracas, Venezuela. 167 pp.

Ramos-Elorduy, J. y Moreno, J.M.P. (2004) Los Coleoptera comestibles de México. Anales del Instituto de Biología, UNAM (Serie Zoología), 75(1): 149-183.

Rodrigues-Netto, S., Campos, T. y Ide, S. (2003) Euchroma gigantea (Linnaeus) (Coleoptera: Buprestidae) como causa da queda de Chorisia speciosa St. Hil. (Bombacaceae). Arquivos do Institu to Biológico, 70: 381-384.

Rosado, L. y Salazar, J. (2005) Coleóptera (III): sobre algunas localidades colombianas para conocer y estudiar a Acrocinus longimanus (L.) y Euchroma gigantea (L.) (Coleoptera: Cerambycidae-Buprestidae). Boletín Científico del Centro de Museos, 9: 139-153.

Ouboter, P., Ouboter, D. y Kadosoe, V. (2020) NZCS Invertebrates Old Collection Before 1987. Version 1.5. National Zoological Collection of Suriname (NZCS). Occurrence dataset. Consultado 20 febrero 2021. https:// doi.org/10.15468/wtwwmz acceso vía GBIF.org. https: / / www.gbif.org/ occurrence/2004970170

Silva, A., Gonçalves, C., Galvão, D., Gonçalves, A., Gomes, J., Silva, M. y Simoni, L. (1968) Quarto catálogo dos insetos que vivem nas plantas do Brasil. Seus parasitos e predadores. Parte II, 1o Tomo. Ministério da Agricultura, Laboratório Central de Patologia Vegetal. Rio de Janeiro. 622 pp.

Shorthouse, D.P. (2010) SimpleMappr an online tool to produce publication-quality point maps. Consultado 6 febrero 2021. Disponible en: https: / www.simplemappr.net.

Ueda, K. (2021) iNaturalist Research-grade Observations. iNaturalist.org. Occurrence dataset. Consultado 20 febrero 2021. https: / / doi.org/10.15468 / ab3s5x acceso vía GBIF. org. https: / / www.gbif.org/occurrence/3031759678 